\title{
An uncommon manifestation of Streptococcus gallolyticus infective endocarditis with cerebral septic emboli
}

\author{
Wan Tin Lim*1, Chiara Jiamin Chong ${ }^{1}$, Robert Chen ${ }^{2}$, Tharmmambal Balakrishnan ${ }^{1}$ \\ ${ }^{1}$ Department of Internal Medicine, Singapore General hospital, Singapore \\ ${ }^{2}$ Department of Diagnostic Radiology, Singapore General hospital, Singapore
}

Received: July 24, 2017

DOI: $10.5430 /$ crim.v4n4p16
Accepted: September 13, $2017 \quad$ Online Published: September 20, 2017

URL: https://doi.org/10.5430/crim.v4n4p16

\begin{abstract}
Infective endocarditis (IE) can often present with neurological manifestations, due to embolization from valvular vegetation, but the presentation is often variable and unpredictable. Septic emboli to both occipital lobes supplied by the posterior cerebral arteries, resulting in visual disturbances are also an uncommon presentation of IE reported in the literature. While S. gallolyticus is a classical cause of IE, it is less common and usually occurs in a less suspecting group of patients with no predisposing cardiac conditions. We report the case of a 48-year-old man, who presented with predominant complaints of blurring of vision and temporal headache, without any other infective symptoms. The procalcitonin level was also normal even in the setting of bacteremia with septic embolism. The initial magnetic resonance imaging (MRI) showed multifocal enhancing lesions in cerebral hemispheres, cerebellum, and brainstem, with leptomeningeal enhancement. Transesophageal echocardiography and blood cultures subsequently confirmed diagnosis of $S$. gallolyticus IE of the mitral valve. The patient was treated with antibiotics upon diagnosis of IE. However, he developed intracranial hemorrhage secondary to mycotic aneurysms, and partial seizures. He eventually succumbed to the intracranial hemorrhage. This case serves to highlight that neurological manifestations can precede symptoms or signs of IE and the presentation are often variable. A high degree of clinical suspicion is needed to suspect neurological manifestations of IE, especially in patients without risk factors.
\end{abstract}

Key Words: Streptococcus gallolyticus, Endocarditis, Ocular, Procalcitonin, Mycotic aneurysm

\section{INTRODUCTION}

Neurological manifestation as the first sign of infective endocarditis (IE) has been reported to have an incidence of $20 \%$ to $40 \%,{ }^{[1,2]}$ but the presentation is often variable and unpredictable. Studies have tried to determine the risk factors associated with neurological complications in IE from embolization and the most predictive tends to be the organisms involved. Staphylococcus auerus, Enterococcus and Streptococcus Viridans are the organisms most likely to develop septic emboli. Our patient presented with an uncommon man- ifestation of Streptococcus gallolyticus IE by Streptococcus gallolyticus with predominant visual disturbances, without any infective signs or symptoms. The infective markers namely the procalcitonin and white cell counts were unremarkable despite the bacteremia and septic embolism. While S. gallolyticus endocarditis is a classical cause of IE, it is less common. ${ }^{[3-6]}$ Embolism to both occipital lobes, which are supplied by the posterior cerebral arteries, is also infrequent, and is the cause of the visual disturbances from lesion of the visual cortex.

*Correspondence: Wan Tin Lim; Email: lim.wan.tin@singhealth.com.sg; Address: Department of Internal Medicine, Singapore General hospital, Singapore. 


\section{CASe presentation}

A 48-year-old Chinese male was admitted for complaints of sudden onset blurring of vision of one week duration. $\mathrm{He}$ described the blurring of vision to be painless and fluctuating. This was associated with mild right temporal headache. There was otherwise no weakness, numbness or facial asymmetry. Further history revealed significant loss of weight of $8 \mathrm{~kg}$ over 6 months, associated with lethargy. He denied any febrile episodes. Prior to hospitalization, he had sought consultation with an Ophthalmologist who had then arranged for a Magnetic Resonance Imaging (MRI) of the brain. The MRI showed multifocal enhancing lesions in cerebral hemispheres, cerebellum, brainstem with leptomeningeal enhancement, and larger lesions in the right posterior-medial temporal lobe. He was then referred to the hospital for further work up and management. His medical history included hypertension and diabetes mellitus that was complicated by peripheral neuropathy.

On examination, vital signs were unremarkable. He was alert and comfortable. The heart, lungs and abdominal examinations were unremarkable. Neurological examination showed power $5 / 5$ in all four limbs, with intact reflexes but a stocking distribution of loss of sensation to pinprick. Cranial nerves examination was intact. In particular, there was no loss in gross visual acuity or visual field defects. Pupils were symmetrical and reactive to light.

\subsection{Investigations \& treatment}

The initial blood investigations showed normocytic normochromic anemia with mild thrombocytopenia. The white blood cell counts, differentials and procalcitonin were not elevated but C-reactive protein was raised at $53 \mathrm{mg} / \mathrm{L}$. Erythrocyte sedimentation rate was $38 \mathrm{~mm} / \mathrm{hr}$ which was unremarkable for Giant cell arteritis. Chest radiography showed mild cardiomegaly and left sided mild pleural effusion. A di- agnostic lumbar puncture to look for infection or malignancy showed clear fluid, 13 white blood cells, 10 red blood cells, glucose $3.7 \mathrm{mmol} / \mathrm{L}$, protein $0.84 \mathrm{~g} / \mathrm{L}$, and the presence of oligoclonal band. Cerebrospinal (CSF) fluid was sent for cultures and TB DNA, which was unyielding for any bacterial, fungal or mycobacterium organism. CSF cytology showed hypercellular yield with predominantly small lymphocytes and other mononuclear cells. CSF flow cytometry also did not show evidence of lymphoproliferative disorders. In particular, there was no obvious enlarged abnormal lymphoid cell seen. Retroviral screen was negative. Computed tomography of the chest, abdomen and pelvis was performed to screen for malignancy but was unremarkable for any masses, and showed only non-specific small to prominent paratracheal, hilar and retroperitoneal lymph nodes. The patient also went on to have an esophagogastroduodenoscopy (OGD) and colonoscopy as part of malignancy workup. OGD revealed only antral gastritis and erosive duodenitis. The colonoscopy was however performed only up to the transverse colon as there was a tight kink in the distal transverse colon. A Formal Goldman Visual Field assessment showed left superior homonymous quadrantanopia. The other eye assessments were otherwise normal.

Transthoracic echocardiogram (TTE) was performed on day four of admission, in view of chest radiography findings suggestive of mild overload, and it incidentally revealed a mobile calcified echo dense mass measuring 13 by $9 \mathrm{~mm}$ at the anterior A3 mitral valve leaflet. Two sets of peripheral blood cultures were performed and streptococcus gallolyticus grew in all culture bottles. The patient was then started on empirical intravenous ceftriaxone, vancomycin and gentamycin for treatment of IE, and later switched to intravenous Benzylpenicillin based on the culture sensitivity. Subsequently, blood cultures showed clearance of bacteremia.

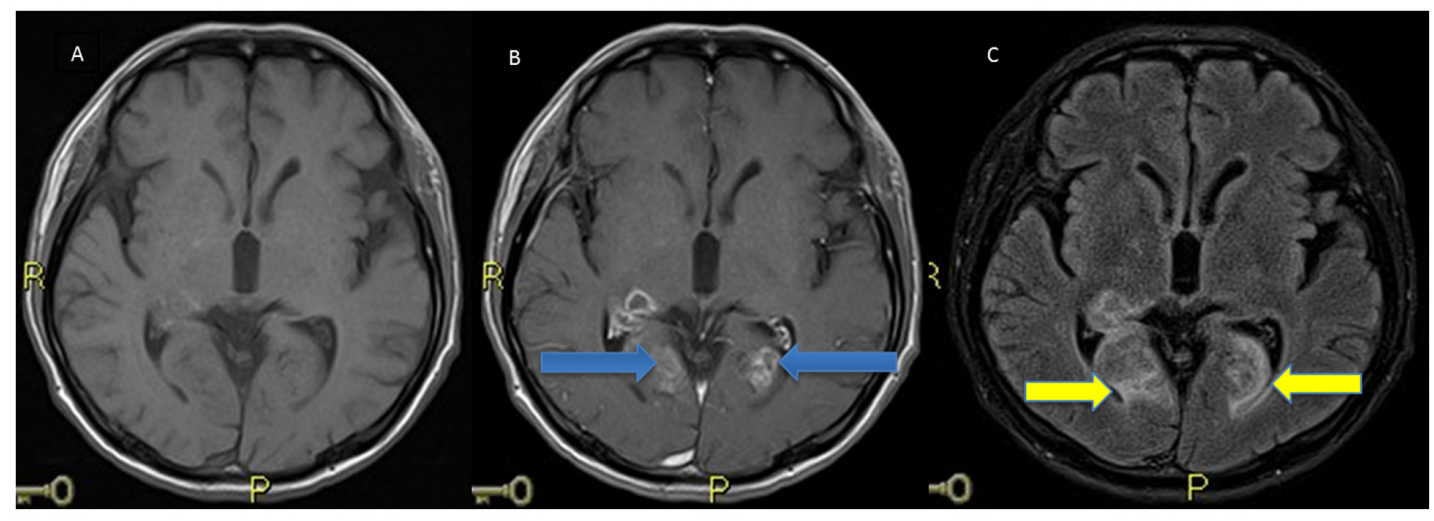

Figure 1. Multisequential Magnetic resonance imaging

A) $T 1$ precontrast image; $B) T 1$ post contrast image demonstrating rim enhancing abscesses in both occipital lobes (blue arrows); $C$ ) FLAIR image showing surrounding vasogenic edema around the abscesses (yellow arrows). 


\subsection{Progress and outcome}

In view of presence of vegetation on echocardiography and his presentation with visual disturbances, MRI brain was repeated. Post contrast T1 image showed several peripherally enhancing lesions within the right medial temporal lobe and bilateral occipital lobes (see Figure 1), with punctate foci of enhancement within both cerebral hemispheres. Compared to the first MRI, it showed stable enhancing lesion in the right occipital lobe but progressive enhancement in the left occipital lobe. Trans-esophageal echocardiogram was performed this time and it demonstrated a stable large filamentous mass attached to the posteromedial commissural segment and its adjacent S3 segment, causing severe and eccentric mitral regurgitation. The cardiac contractility was otherwise preserved. At this point in time, it has become clear that the diagnosis was disseminated intracranial lesions secondary to septic emboli from $S$. gallolyticus IE.
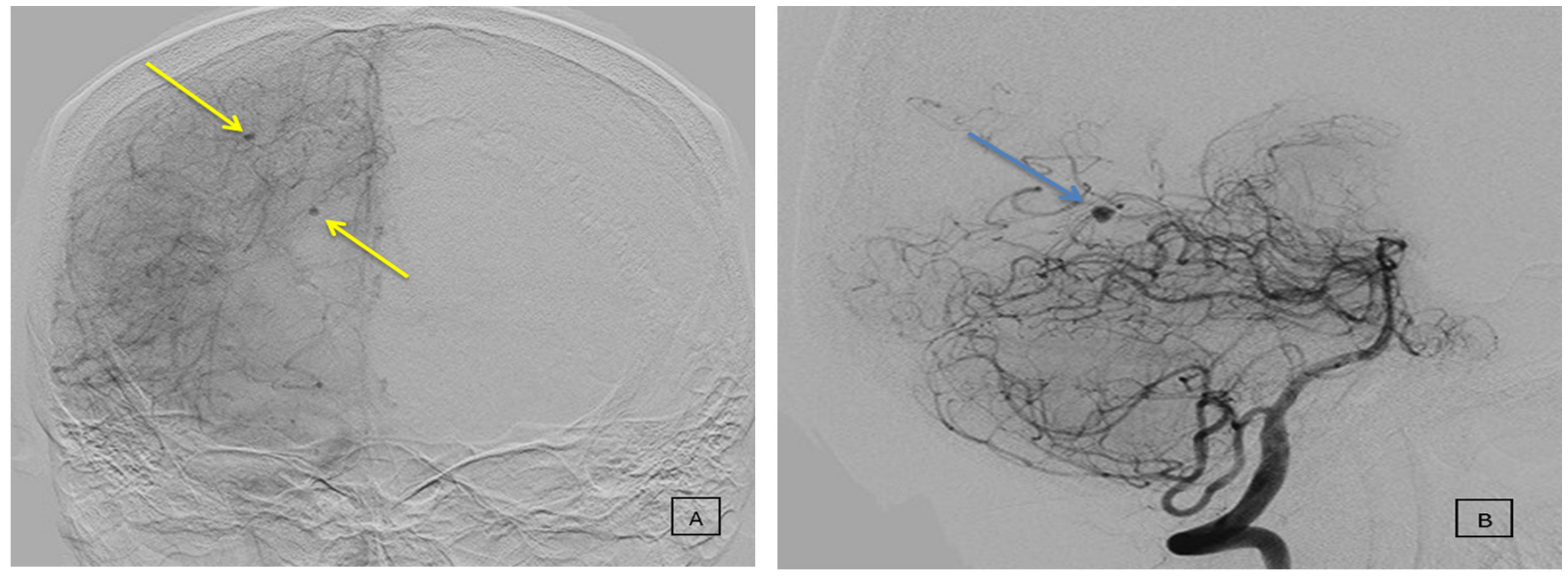

Figure 2. Diagnostic angiogram demonstrates multiple small mycotic aneurysms within the (A) anterior (yellow arrows) and (B) posterior circulations (blue arrow)

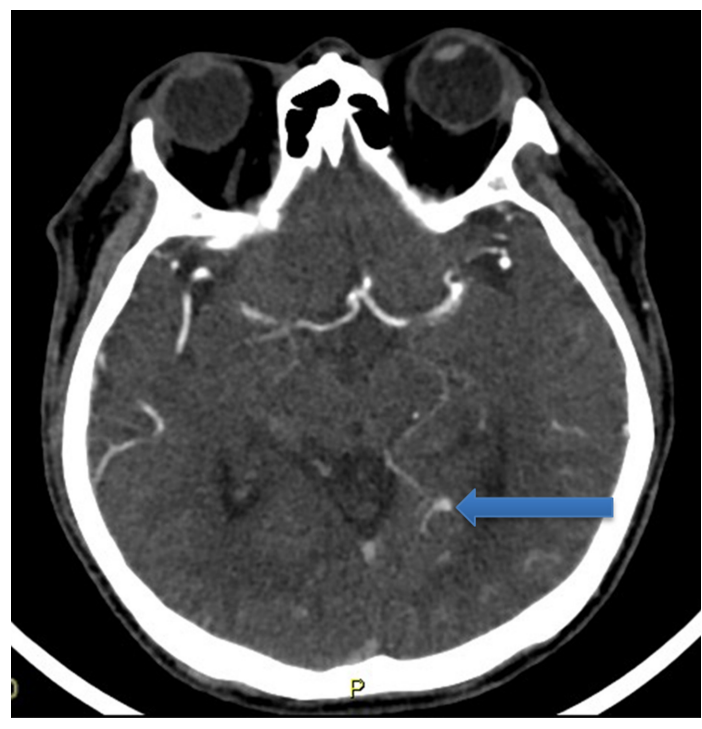

Figure 3. CT Angiogram demonstrates a small mycotic aneurysm arising from the distal left posterior cerebral artery in the $\mathrm{P} 4$ division

Two weeks after initiation of antibiotics, he complained of two episodes of left upper limb jerking which lasted for a few minutes, with residual weakness after each episode. He had no loss of consciousness during the two episodes of jerking movements. An urgent CT brain was performed which showed an acute right frontal and left cerebellar hemorrhage. A diagnostic angiography of the cerebral vessels performed showed several intracranial aneurysms (see Figure 2), with a small aneurysm arising from a distal branch of the left middle cerebral artery and a larger $3.5 \mathrm{~mm}$ aneurysm arising from the left posterior cerebral artery (see Figure 3). One of the aneurysms lay within the acute right frontal hematoma which was the likely cause of the bleed.

In view of the widespread multiple mycotic aneurysms that are not surgically treatable without causing further ischemia to the brain, a decision was made to adopt a conservative approach. A few days later, there was a drop in his Glasgow Coma Scale (GCS) score and repeat CT brain then showed significant bleed in the left cerebral hemisphere with mass effect and evolution of the right frontal hematoma. In view of the extent of the intracranial bleed with guarded prognosis and poor outcome, he was managed conservatively with comfort measures. He subsequently demised the next day.

\section{Discussion}

Recent studies have suggested a new nomenclature for S. bovis, so that $S$. bovis biotype I is now named $S$. gallolyticus. ${ }^{[7]}$ S. gallolyticus endocarditis is a classical, but less common cause of IE, even though it is rising in incidence in the re- 
cent years. More common causative pathogens remain to be Staphylococcus aureus and Streptococcal viridans. ${ }^{[3-5]}$ The incidence of $S$. gallolyticus varies according to geographical location. The international collaboration study on IE was a prospective cohort study that evaluated 2,781 cases of definite IE in adults from 2000-2005. It reported an incidence of S. gallolyticus as follows: $2 \%$ (9/597) in North America, 7\% (17/254) in South America, 10\% (116/1,213) in Europe, and 3\% (23/717) in Africa, Middle East and Asia combined. ${ }^{[8]}$ Risk factors associated with early embolization has often been an interest of study, in order to better anticipate and diagnose embolic diseases early. S. bovis I infection was found to be associated with advanced age, with more frequent involvement of native valves, and a higher rate of multiple embolic events. ${ }^{[8,9]}$

The association of $S$. gallolyticus IE and colonic lesions has long been established. Recent studies have also found the association of $S$. gallolyticus IE with inflammatory bowel disease and chronic liver disease. ${ }^{[8]}$ It was found that $25 \%$ to $80 \%$ of patients with S. gallolyticus bacteremia and $18 \%$ to $62 \%$ of patients with $S$. gallolyticus endocarditis have underlying colorectal tumors. ${ }^{[10-17]}$ In view of our patient's history of loss of weight and incomplete colonoscopy, it was possible that he had an underlying undiagnosed colonic tumor.

Our patient presented with main complaint of intermittent blurring of vision associated with headache as a manifestation of cerebral embolization, with no associated infective symptoms, signs or cardiac risk factor of IE. The infective markers were also unremarkable. As such, the diagnosis of IE was made much later. Procalcitonin has been known for its high sensitivity and diagnostic accuracy for differentiating bacterial from viral infections. ${ }^{[18]}$ Various studies have also investigated the diagnostic values of serum procalcitonin in IE. The studies suggest that procalcitonin was significantly higher in patients with IE with bacteremia due to endocarditis-typical organisms being the strongest independent determinant of high procalcitonin. ${ }^{[19-22]}$ However, one study showed that IE patients with Staphylococcus aureus bacteremia had significantly high procalcitonin levels, but surprisingly much lower procalcitonin in bacteremia other than Staphylococcus aureus. ${ }^{[21]}$ This might explain why in our case, procalcitonin remained completely normal despite patient being bacteremic with septic embolism. However, further studies are needed to verify this finding. C-reactive protein and sedimentation rate are not proven to have correlation with diagnosis of IE. ${ }^{[23]}$

IE with cerebrovascular complications has always been a diagnostic, therapeutic and prognostic challenge. The frequent
MR imaging findings in neurological complications in IE are often acute ischemic lesions in watershed territories of varying ages and cerebral microbleeds distributed in cortical areas. ${ }^{[2]}$ There has been studies suggesting that there might be a role in using the pattern of ischemic lesions and cerebral microbleeds as a further diagnostic marker to aid in making the diagnosis of IE. ${ }^{[24-26]}$ Watershed strokes are localized to either the cortical or internal watershed areas, of which involvement of the cortical branches of the posterior cerebral artery territory can lead to presentation of visual loss with upper quadrantic homonymous hemianopia. While most of the cerebrovascular complications are symptomatic, ${ }^{[1,27-30]}$ silent cerebral complications were reported to be as high as $30 \%$ of patients with left-sided IE, as reported in a prospective study by Snygg et al. ${ }^{[1]}$ The most frequent neurologic manifestations are non-specific headache, focal neurological deficits, encephalopathy, meningism or seizure, transient ischemic attack, ischemic stroke, intracerebral hemorrhage or meningitis. Presentation depends on the location of cerebra-embolization. The onset of neurological symptoms also varies, with some patients reporting symptoms on presentation, and others manifesting neurological symptoms only after the start of treatment. Visual symptoms were rarely mentioned in the literature. In 1967, Harrison and Hampton ${ }^{[27]}$ reported a case series of patients with bacterial endocarditis where four out of 23 patients with neurological complaints had visual symptoms. Our case presented with fluctuating blurring of vision which is likely a manifestation of transient ischemic attack related to cerebra-embolization. This case highlights the importance of recognizing visual disturbances as a manifestation of this disease entity, especially in patients without risk factors of IE and in the absence of raised inflammatory parameters. More systematic studies of cerebral leison associated with endocarditis can be done to better characterize this association, for earlier diagnosis to be made.

\section{Conclusion}

S. gallolyticus IE is not common, but is commonly associated with advanced age, native valve IE with increased rate of embolization event and gastrointestinal diseases such as colonic tumors, inflammatory bowel disease and liver diseases. Visual disturbance can be the only initial presentation of IE which can be protean in nature; as such physicians need to consider IE as a possible diagnosis in patients, even if there are no predisposing cardiac risk factors and unremarkable infective markers.

\section{CONFlicts OF InTEREST Disclosure}

The authors have declared no conflicts of interest. 


\section{REFERENCES}

[1] Snygg-Martin U, Gustafsson L, Rosengren L, et al. Cerebrovascular complications in patients with left-sided infective endocarditis are common: a prospective study using magnetic resonance imaging and neurochemical brain damage markers. Clin Infect Dis. 2008; 47(1): 23-30. PMid:18491965 https ://doi .org/10.1086/588663

[2] Heiro M, Nikoskelainen J, Engblom E, et al. Neurologic manifestations of infective endocarditis: A 17-year experience in a teaching hospital in Finland. Arch Intern Med. 2000; 160(18): 2781-7. PMid:11025788 https://doi.org/10.1001/archinte.160.1 8.2781

[3] Lockrow J, Longstreth W, Davis AP. Intracranial Aneurysms From Presumed Infective Endocarditis: The Dilemma of Persistently Negative Cultures. Neurohospitalist. 2016; 6(2): 80-6. PMid:27053986 https://doi.org/10.1177/1941874415605879

[4] Slipczuk L, Codolosa JN, Davila CD, et al. Infective endocarditis epidemiology over five decades: A systematic review. PLoS One. 2013; 8(12). PMid:24349331 https://doi.org/10.1371/jour nal. pone. 0082665

[5] Vogkou CT, Vlachogiannis NI, Palaiodimos L, et al. The causative agents in infective endocarditis: a systematic review comprising 33,214 cases. Eur J Clin Microbiol Infect Dis. 2016: 1-19. https : //doi.org/10.1007/s10096-016-2660-6

[6] Mello R, da Silva Santos M, Golebiosvki W, et al. Streptococcus bovis endocarditis: Analysis of cases between 2005 and 2014. Brazilian J Infect Dis. 2015; 19(2): 209-12. PMid:25636194 https: //doi.org/10.1016/j.bjid.2014.12.007

[7] Beck M, Frodl R, Funke G. Comprehensive study of strains previously designated Streptococcus bovis consecutively isolated from human blood cultures and emended description of Streptococcus gallolyticus and Streptococcus infantarius subsp. coli. J Clin Microbiol. 2008; 46(9): 2966-72. PMid:18614655 https://doi.org/10.1 128/JCM. 00078-08

[8] Tripodi MF, Adinolfi LE, Ragone E, et al. Streptococcus bovis Endocarditis and its Association with Chronic Liver Disease: an Underestimated Risk Factor. Clin Infect Dis. 2004; 38(10): 1394-400. PMid:15156477 https://doi.org/10.1086/392503

[9] Duval X, Papastamopoulos V, Longuet P, et al. Definite Streptococcus bovis endocarditis: Characteristics in 20 patients. Clin Microbiol Infect. 2001; 7(1): 3-10. PMid:11284936 https ://doi .org/10.1 046/j.1469-0691.2001.00190.x

[10] Gupta A, Madani R, Mukhtar H. Streptococcus bovis endocarditis, a silent sign for colonic tumour. Color Dis. 2010; 12(3): 164-71. PMid:19226366 https://doi.org/10.1111/j.1463-1318.20 $09.01814 . \mathrm{x}$

[11] Abdulamir AS, Hafidh RR, Abu Bakar F. The association of Streptococcus bovis/gallolyticus with colorectal tumors: the nature and the underlying mechanisms of its etiological role. J Exp Clin Cancer Res. 2011; 30(1): 11. PMid:21247505 https ://doi.org/10.1186/17 56-9966-30-11

[12] Kok H, Jureen R, Soon CY, et al. Colon cancer presenting as Streptococcus gallolyticus infective endocarditis. Singapore Med J. 2007; 48(2): e43-5. PMid:17304377

[13] Leport C, Bure A, Leport J, et al. Incidence of Colonic Lesions in Streptococcus Bovis and Enterococcal Endocarditis. Lancet. 1987; 329(8535): 748. https://doi.org/10.1016/S0140-6736(87 ) $90391-6$

[14] Malkin J, Kimmitt PT, Ou HY, et al. Identification of Streptococcus gallolyticus subsp. macedonicus as the etiological agent in a case of culture-negative multivalve infective endocarditis by $16 \mathrm{~S}$ rDNA PCR analysis of resected valvular tissue. J Heart Valve Dis. 2008; 17(5): 589-92. PMid:18980096
[15] Friedman E, Elian D, Eisenstein Z. Streptococcus bovis bacteremia and underlying gastrointestinal neoplasms. Med Pediatr Oncol. 1986; 14(6): 313-5. PMid:3784983 https://doi.org/10.1002/mpo. 2950140606

[16] Reynolds JG, Silva E, McCormack WM. Association of Streptococcus bovis bacteremia with bowel disease. J Clin Microbiol. 1983; 17(4): 696-7. PMid:6853693

[17] Zarkin BA, Lillemoe KD, Cameron JL, et al. The triad of Streptococcus bovis bacteremia, colonic pathology, and liver disease. Ann Surg. 1990; 211(6): 786-91-2.

[18] Simon L, Gauvin F, Amre DK, et al. Serum procalcitonin and Creactive protein levels as markers of bacterial infection: A systematic review and meta-analysis. Clin Infect Dis. 2004; 39(2): 206-17. PMid:15307030 https : //doi.org/10.1086/421997

[19] Knudsen JB, Fuursted K, Petersen E, et al. Procalcitonin in 759 patients clinically suspected of infective endocarditis. Am J Med. 2010; 123(12): 1121-7. PMid:20870199 https ://doi .org/10.1016/j . amjmed.2010.07.018

[20] Kocazeybek B, Küçükoğlu S, Oner YA. Procalcitonin and C-reactive protein in infective endocarditis: Correlation with etiology and prognosis. Chemotherapy. 2003; 49(1-2): 76-84. PMid:12714816 https://doi.org/10.1159/000069777

[21] Cuculi F, Toggweiler S, Auer M, et al. Serum procalcitonin has the potential to identify Staphylococcus aureus endocarditis. Eur J Clin Microbiol \& Infect Dis. 2008; 27(11): 1145-9. PMid:18521635 https ://doi.org/10.1007/s10096-008-0541-3

[22] Mueller C, Huber P, Laifer G, et al. Procalcitonin and the Early Diagnosis of Infective Endocarditis. Circulation. 2004; 109(14): 1707-10. PMid:15066945 https ://doi.org/10.1161/01.CIR.0000126 281.52345 .52

[23] Gouriet F, Bothelo-Nevers E, Coulibaly B, et al. Evaluation of sedimentation rate, rheumatoid factor, $\mathrm{C}$-reactive protein, and tumor necrosis factor for the diagnosis of infective endocarditis. Clin Vaccine Immunol. 2006; 13(2): 301. PMid:16467342 https ://doi .or g/10.1128/CVI.13.2.301.2006

[24] Hess A, Klein I, Iung B, et al. Brain MRI findings in neurologically asymptomatic patients with infective endocarditis. AJNR Am J Neuroradiol. 2013; 34(8): 1579-84. PMid:23639563 https : //doi.org/10.3174/ajnr.A3582

[25] Klein I, Iung B, Labreuche J, et al. Cerebral microbleeds are frequent in infective endocarditis: A case-control study. Stroke. 2009; 40(11): 3461-5. PMid:19762695 https://doi.org/10.1161/STROKEAH A. 109.562546

[26] Duval X. Effect of Early Cerebral Magnetic Resonance Imaging on Clinical Decisions in Infective Endocarditis. Ann Intern Med. 2010; 152(8): 497. PMid:20404380 https ://doi.org/10.7326/0003 -4819-152-8-201004200-00006

[27] Thuny F, Tribouilloy C, Giorgi R, et al. Impact of cerebrovascular complications on mortality and neurologic outcome during infective endocarditis: a prospective multicentre study. 2007: 1155-61.

[28] Castilla-Guerra L, Fernández-Moreno MC, Fernández-Bolanos Porras R, et al. Neurologic manifestations of infective endocarditis: A diagnostic and prognostic challenge. Rev Neurol. 2011; 41(10): 577 81 .

[29] Kanter MC, Hart RG. Neurologic complications of infective endocarditis. Neurology. 1991; 41(7): 1015-20. https ://doi.org/10 $.1212 /$ WNL . 41.7 .1015

[30] Jones HR, Siekert RG. Neurological manifestations of infective endocarditis. Review of clinical and therapeutic challenges. Brain. 1989 October: 1295-315. PMid:2679968 https ://doi.org/10.1093/ brain/112.5.1295 